\title{
Sample containerization and planetary protection using brazing for breaking the chain of contact to Mars
}

Yoseph Bar-Cohen, Mircea Badescu, Stewart Sherrit, Xiaoqi Bao, Hyeong Jae Lee, et al.

Yoseph Bar-Cohen, Mircea Badescu, Stewart Sherrit, Xiaoqi Bao, Hyeong Jae Lee, Erik Bombela, Sukhwinder Sandhu, "Sample containerization and planetary protection using brazing for breaking the chain of contact to Mars ," Proc. SPIE 10968, Behavior and Mechanics of Multifunctional Materials XIII, 1096802 (29 March 2019); doi: 10.1117/12.2513969

Event: SPIE Smart Structures + Nondestructive Evaluation, 2019, Denver, Colorado, United States 


\title{
Sample containerization and planetary protection using brazing for breaking the chain of contact to Mars
}

\author{
Yoseph Bar-Cohen, Mircea Badescu, Stewart Sherrit, Xiaoqi Bao, Hyeong Jae Lee, Erik Bombela \\ and Sukhwinder Sandhu \\ Jet Propulsion Laboratory, California Institute of Technology, 4800 Oak Grove Drive, Pasadena, CA \\ 91109-8099, yosi@jpl.nasa.gov, web: http://ndeaa.jpl.nasa.gov
}

\begin{abstract}
Returning samples from Mars to Earth in a future NASA mission would require protection of our planet from the potential risks of bringing uncontrolled biological materials back with the samples. Part of the planetary protection process would involve a process called "breaking the chain of contact (BTC)", where any returned material reaching Earth for further analysis would have to be sealed inside a container at extremely high confidence. The sterilization process would require destroying any potential biological materials that may contaminate the external surface of the container. A novel process for containing returning samples has been conceived and is under development at JPL. The process consists of using induction heated brazing to synchronously sterilize, separate, seam and seal the container. The braze material is heated to the melting temperature that is higher than $500^{\circ} \mathrm{C}$ and, thus, it assures sterilizing exposed areas since all carbon bonds are broken above this temperature. The container consists of double walls with "Earth clean" interstitial space. The process consists of two-steps, where one is intended to be prepared on Earth and the second would be executed on orbit around Mars. The latest results of this study will be described and discussed.
\end{abstract}

Keywords: Sample return; planetary exploration; induction heated brazing; synchronous Sterilization, Sealing, Separation and Seaming (S4); break the chain (BTC); containerization; and planetary protection.

\subsection{Introduction}

One of the key goals of the NASA exploration missions is to determine if there is life elsewhere in the Universe, namely answer the question "are we alone?". This objective is being addressed by remote observations, landed missions that include in-situ sampling and testing, and sample return and testing. In order to maximize the extracted science, the Planetary Science Decadal Survey prioritized returning samples (from such bodies as Mars) back to Earth [1]. Returning samples back to Earth poses a backward contamination risk and requires protecting our planet from potential hazard. Strict protection requirements dictated probability of failure of containment that is not more than $10^{-6}$ for a $50 \mathrm{~nm}$ particle, where higher probability would lead to aborting the mission and not returning the sample container [2]. To meet the required protection, it is necessary to "break the chain of contact (BTC)", where any material reaching Earth would have to be inside a sealed container that is not contaminated externally with martian material at an extremely high level of confidence. Supporting potential sample return missions from potentially habitable environment requires extremely reliable mechanism to assure a very high level of planetary protection of Earth. The mechanism has to provide a hermetic seal of the returned sample container, sterilizing the sealed/seamed section, and preventing biocontaminations from exposing the external surfaces of the returned container.

To prepare for returning samples from Mars, a novel mechanism of containerization and planetary protection has been developed and demonstrated that allows synchronously sterilizing, sealing, separating and seaming (S4) the container that would enclose returned samples [3 - 6]. The mechanism involves induction heated brazing [7], and the prototype container consists of double walls having equivalent of "Earth clean" interstitial surfaces. The execution of the containerization mechanism is done in two steps that are equivalent to preparation on Earth and finalizing on orbit around the sampled body in potential future missions. In the latter step, the samples are placed inside the inner section of the protection container and melting the braze at temperatures higher than $500^{\circ} \mathrm{C}$ to assure sterilization of the exposed interface section. At this temperature and above, carbon bonds break via pyrolysis destroying any potential presence of biological material. The steps of this BTC brazing process are as follows:

- Step-1: Executed on Earth - the double wall container halves are fabricated and brazed.

Behavior and Mechanics of Multifunctional Materials XIII, edited by Hani E. Naguib, Proc. of SPIE Vol. 10968, 1096802 · @ 2019 SPIE · CCC code: 0277-786X/19/\$18 · doi: 10.1117/12.2513969 
- Step-2: Executed on orbit - the Orbiting Samples (OS) are inserted into the inner container section, the pre-brazed joints are melted and new braze joints are formed in the $\mathrm{S} 4$ process.

In this manuscript, the progress in this ongoing study is described and discussed.

\subsection{Modeling induction heated brazing of the BTC container}

Designing the BTC containerization mechanism requires analytically modeling the brazing process and finite element (FE) models have been used to numerically analyze and simulate various configurations. Potential designs of prototype containers that are made of stainless steel and titanium were analyzed and the temperature rise at various locations have been determined. The analysis involved parametric studies of designs with better temperature control and lower power consumption. A generic BTC container configuration that is subjected to induction heating at zero-gravity (simulating being on orbit around Mars) is shown in Figure 1. To simulate the induction heated brazing process, multi-frame FE modeling has been done that combines the thermal and electromagnetic models.

A multi-physics FE analysis including induction heating and thermal models was performed to simulate the S4 process in support of the concept designs. The configuration consists of a hemispherical lower shell, which is a part of the wall of the spacecraft and pre-brazed to the base structure on Earth forming one double wall structure. Separately, the Cap and the Top section of the shell are pre-brazed together on Earth forming the second double wall assembly. The inside space of the double wall assembly is Earth-clean (pre-exposed only to Earth's environment) and it is the lower section in the Figure. The samples that are inside the tube assemblies are fixed and enclosed in a spherical shell that is potentially contaminated with Mars dust on the external surface. A close-up view of the joint area is shown in Figure 1 right with details of the area where the brazing and separation of the shell from the Cap and Base are performed. The two vertical interfaces are the pre-brazed joints that would be separated. The oblique interfaces are those that would be seamed. The shell of the OS (samples enclosure) is made of aluminum with tentative diameter of $27 \mathrm{~cm}$. The BTC container (double walls) are made of stainless steel 304 or titanium alloy Ti-6AL-4V. The temperature dependent electric and thermal properties used in the simulation have been based on numbers that were taken from the open literature. A simplified model is applied to the OS (sample tube assemblies) represented by three cylindrical tubes with homogenous material having equivalent thermal properties. A high temperature insulation material made of ceramic foam (MIN-K) is selected for the insulation layer between the BTC shell and the OS. The melting point of the brazing material is set at $750^{\circ} \mathrm{C}$. In addition to the heat conduction in the solid elements, the thermal model includes the heat radiation within the enclosure of the Cap-BTC top shell and from the outer surfaces to ambient.

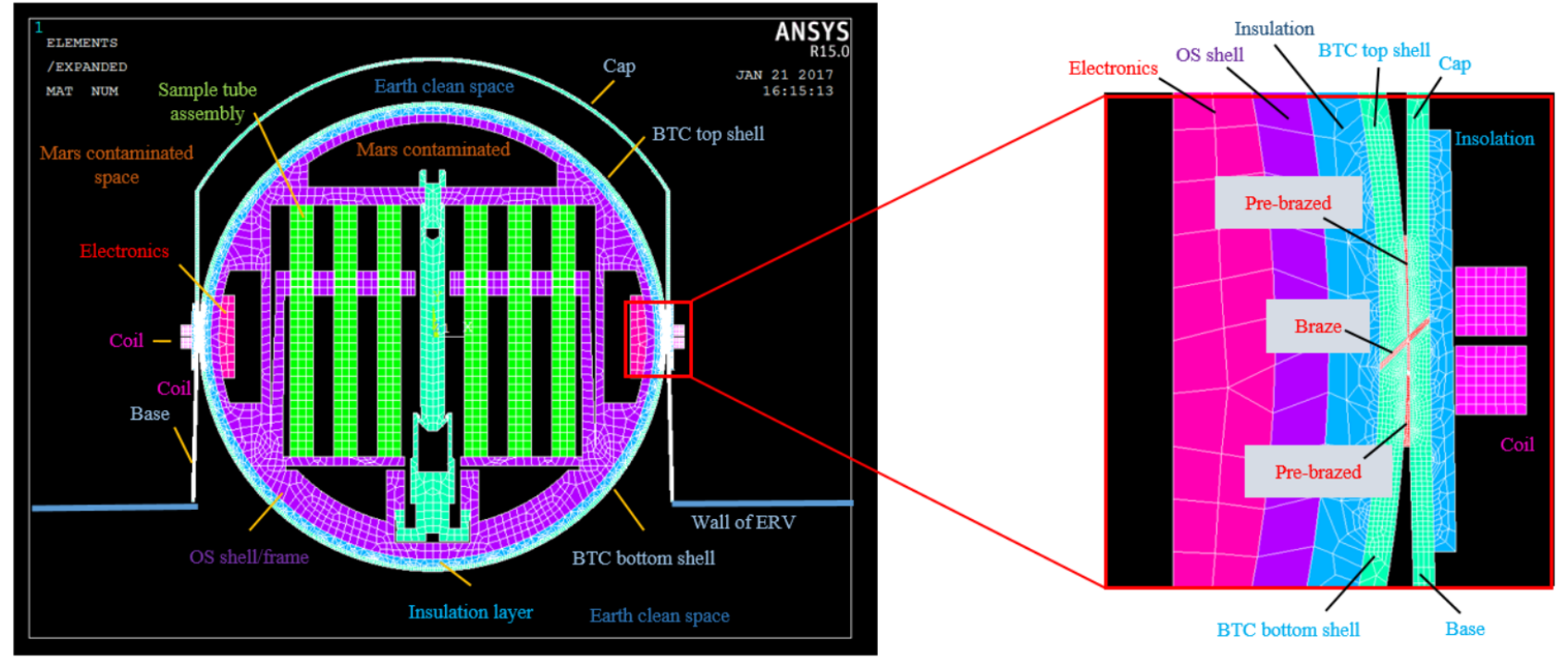

Figure 1: FE thermal model of a concept design for the BTC using induction heating. The BTC shell, cap and base are made of Ti-6Al-4V or SS304. The insulation layer is assumed to be made of ceramic foam MIN-K. 
The electromagnetic FE model simulates the induction heating and it is similar to the thermal model described above. For simplicity, the detailed structures inside the OS (aluminum shell of the sample enclosure) were omitted because the electromagnetic field is expected to be very weak inside due to the shielding effect of the metallic shells in the frequency range of interest $(20-120 \mathrm{kHz})$. This simplification was validated by preliminary simulations of SS304 BTC shell at $0^{\circ} \mathrm{C}$. A 2-turn copper coil with a cross section of $6.5 \times 6.5 \mathrm{~mm}^{2}$ is placed close to the brazing region, the gap between the coil and the BTC wall surface is $5 \mathrm{~mm}$, and the spacing of the two turns is $1 \mathrm{~mm}$.

The two models run alternatively to mimic the mechanism and the material properties are updated to account for the highly temperature dependent properties. The combined models were applied to both SS304 and Ti-6Al-4V BTC shells and temperature dependent thermal and electrical material properties were applied. At the beginning of the simulation, $0^{\circ} \mathrm{C}$ is the assumed ambient temperature and the temperatures of all the components. The temperature of the coil is assumed to be well controlled by a cooling method so that its rise in the coils is neglected and the brazing temperature is $750^{\circ} \mathrm{C}$. Using the model, it was determined that, for the SS304 shells, 420 seconds ( 7 minutes) are required for all the braze material to reach or exceed the brazing temperature of $750^{\circ} \mathrm{C}$ when $480 \mathrm{~A}$ current at $50 \mathrm{kHz}$ is applied to the coil. The total energy consumption is $2000 \mathrm{~kJ}$ with the major heat energy is limited to the zone surrounding the heated area with very small temperature rise taking place inside the OS. The input electric power is from $4.26 \mathrm{~kW}$ at the beginning and rises to $5.30 \mathrm{~kW}$ at 420 second. The total energy consumed after $420 \mathrm{~s}$ are $2108 \mathrm{~kJ}, 1729 \mathrm{~kJ}$ and $379 \mathrm{~kJ}$ for the electrical input, heating and coil loss, respectively. The average coil efficiency is $82 \%$. According to the temperature rise curve predicted in the simulation, the heating step of the simulated process is set to last for 420 seconds. Then, the BTC container would be separated from the Cap-Base, and moved into Earth Return Vehicle (ERV) and allowed to cool down. In the simulation, the container is assumed to be moved to a position far away from the heated Cap-Base area and cools down by radiation loss at the $0^{\circ} \mathrm{C}$ ambient environment. The temperature first rises and then falls after reaching maximum value. The maximum temperature in the sample assemblies is found to be $28.7^{\circ} \mathrm{C}$, which is an acceptable temperature rise for sample stability.

The simulation was also performed for Ti-6Al-4V BTC shell under the same assumptions except for a smaller electric current of 370A being applied. The comparison of the two cases is summarized in Table 1. The analysis suggests the use of the titanium alloy instead of stainless steel significantly decreases the required power and energy and lowers the sample temperature rise.

Table 1: Comparison of the stainless steel and titanium alloy base materials

\begin{tabular}{|c|c|c|c|c|c|c|c|}
\hline Material & $\begin{array}{c}\text { Input } \\
\text { current }\end{array}$ & Input power & $\begin{array}{c}\text { Average } \\
\text { power }\end{array}$ & $\begin{array}{c}\text { Coil } \\
\text { efficiency }\end{array}$ & $\begin{array}{c}\text { Heating } \\
\text { time }\end{array}$ & Energy & Sample temperature \\
\hline & $\mathrm{A}$ & $\mathrm{kW}$ & $\mathrm{kW}$ & $\%$ & $\mathrm{~s}$ & $\mathrm{~kJ}$ & ${ }^{\circ} \mathrm{C}$ \\
\hline $\mathrm{SS}$ & 480 & $4.26-5.30$ & 5.20 & 82.01 & 420 & 2108 & 28.7 \\
\hline $\mathrm{Ti}$ & 370 & $2.68-3.66$ & 3.39 & 84.37 & 326 & 1105 & 16.7 \\
\hline
\end{tabular}

\subsection{The current container configuration}

Developing the BTC brazing S4 process was initially investigated for execution on the surface of Mars. The container was assumed to have a coffee-cup size with cylindrical shape. The details of the developed processes and mechanisms were covered in two NASA New Technology Reports [3 - 4]. As stated earlier, a double wall container having internal gap simulating "Earth clean" volume has been used. A brazing material seams the two halves of the inner container encapsulating the returned samples and they are separated from the two half sections of the external container which effectively becomes part of the lander. The double wall container is prepared on Earth as two separate components: the lid and the base, both having double wall or shells. The acquired samples are inserted into the lid inner shell and the lid is brought in contact with the base section. The outer shell of the base component is part of the lander structure. The inner shells are lined to the inside with a thermally insulating layer for protecting the samples. Inductively heating the brazing material leads to joining the inner shells separately from the outer shells and the brazing materials between the two components are melted separating the formed inner container and sterilizes the joints area. The inner shells are then separated from the outer shells while being held in contact, the heating is stopped and the joints are allowed to cool down. 
Thus, the lid outer shell is brazed to the base outer shell creating a separation wall between the contaminated area and the Earth clean area inside the lander. The inner shells are brazed forming an encapsulated clean container that encloses the samples. The newly formed container located inside the clean area of the lander can now be transferred to the Earth return vehicle for further processing.

The S4 process was demonstrated in a vacuum chamber and the induction heating was done by inserting the container assembly inside the coil that is connected via feed-through to the drive electronics. To minimize oxidation, once the vacuum reaches a level of milli-Torrs, research grade Argon with $99.99 \%$ purity was used to back-fill the chamber and the vacuum pumping is resumed. A successful sealing and separation process was demonstrated and the verification of the sealing was done using a spectrometer based He leak detector by creating a vacuum inside the container connected to the spectrometer and spraying helium on the outside next to the separated and seamed interface section [6].

During the process of heating, the forming container was mounted on a support fixture using a bottom screw for aligning the brazed section with the center plane of the coil. In addition, a retaining bar was placed above the container to keep the inner container from uncontrolled ejection during the melting of the braze material. The assembly was monitored using an infrared camera and was heated until the brazing material melted. The process was stopped when the formed container (seamed lid and the inner section) were ejected by the force of the spring located between the inner and outer lid shells.

The key challenge that was identified when using the cylindrical configuration has been the high likelihood of jamming when inserting the double wall lid into the base. This risk has been addressed by extremely careful insertion when the process was done manually. However, this autonomous insertion is expected to be a great challenge for a robotic mechanism on-orbit. To address this jamming concern, a cone-within-cone design was conceived [6]. Here, the open edge of the double wall container at the lid and the base, has an cone shape interface. The lid and base cone angles match each other and they are used to guide the double wall lid when it is brought in contact with the base for precision alignment. A spring between the outer and inner shells of the double wall lid keeps the lid inner shell preloaded against the base inner shell. After the induction coil is activated and the brazing materials have melted, a separation takes place that is controlled by an active element such as an actuator or a passive element with a timer. The newly formed container encloses the sample containment vessel but continues to be held in place until the joint, which is formed along the conical surface, solidifies. The separation of the container is done along a second conical shaped surface oriented opposite to the initial conical surface such that it allows for the movement of the container axially away from the coil. This increases the radial gap between the moving parts and, therefore, preventing the possibility of parts jamming. The process steps consist of the following: approach, contact, coil activation, separation, coil deactivation and cooling (Figure 2). The angles of the two cones and the number of the brazing rings can be chosen based on space availability and the need to use a smaller or larger volume of braze material.

As opposed to the initial configuration of a coffee-cup, the sample enclosure has evolved to sphere and the execution to be done on orbit around Mars. Using various lessons learned, the BTC brazing process has evolved to what is shown graphically in Figure 3 where the Step-1 prepared components are shown in Figure 3a. The Step-2 process takes place after the capture and enclosing the sample enclosure and the activation of the induction heater to make the braze seal/seam and the melting of the separation braze rings. Successful demonstration of the cone-withincone configuration has been done where a set of parts have been brazed and tested leak-free using spectrometer, vacuum and helium spray as described earlier. In one of the tests, even though the seal was successful, during the S4 process of brazing the prototype container that is shown in Figure 3, the lid inner shell shifted from the axis of the container. To address this issue, a registration feature was added to the conical interfaces of the lid and base and the process was repeated. Again, the container that was subjected to the two steps process was found leak free. In addition, the registration features produced a successful alignment. 
1.

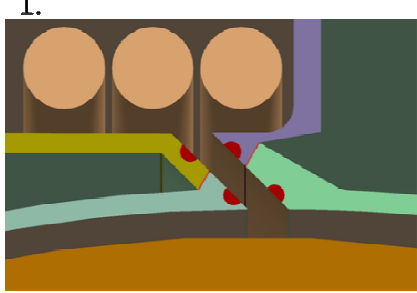

3.

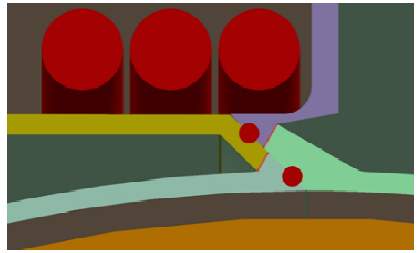

4.

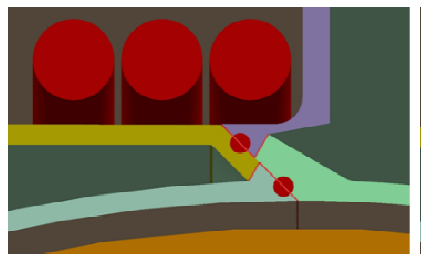

2.

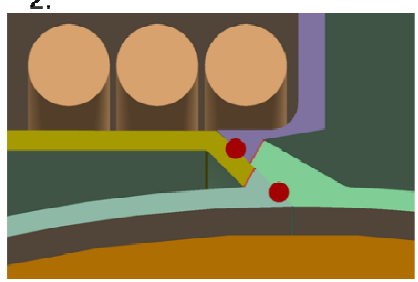

5.

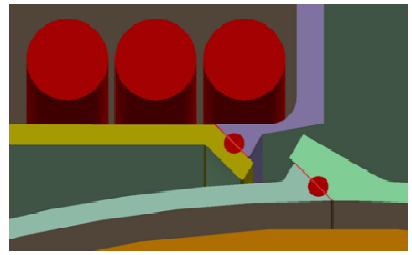

Figure 2: The S4 process steps and the double cone-within-cone representation.

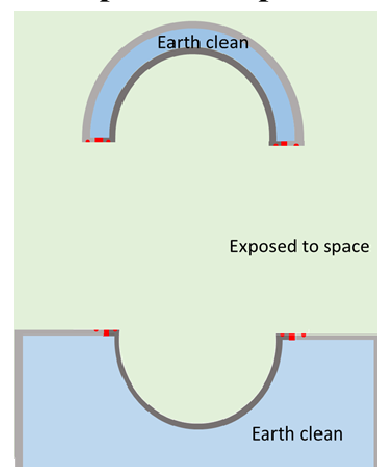

a. In space

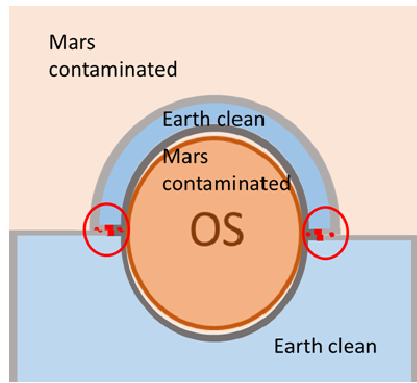

d. Activated heating

(Sterilizing and Sealing)

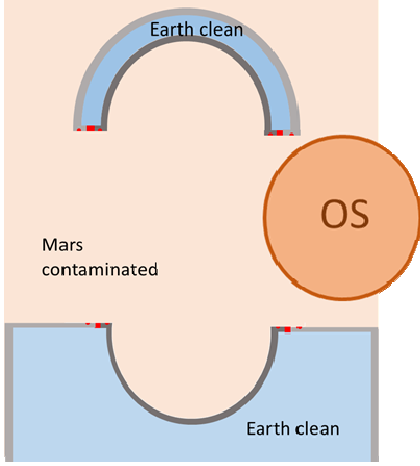

b. OS capture

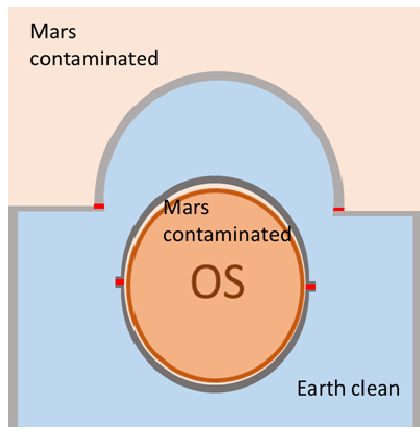

e. Separation

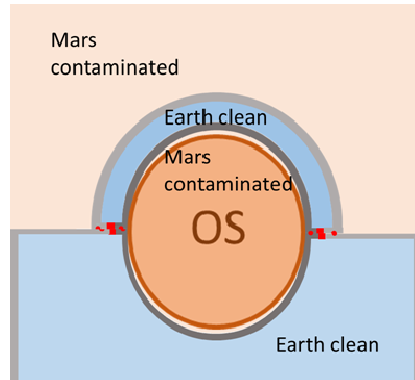

c. Enclose OS

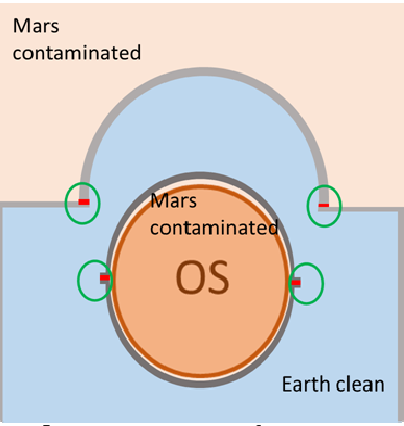

f. Passive cooling

(Seaming)

Figure 3: The Step-2 process of synchronous Sterilization, Sealing, Separation, and Seaming on-orbit

\subsection{The full scale development}

As the plans for the potential Mars Sample Return have evolved, the size of the notional container has been modified to as large as $27 \mathrm{~cm}$ diameter. Upon landing of the Mars 2020 mission, sample cores will be acquired and encased in tubes 
and will be placed in a known location. These tubes containing the core samples are supposed to be acquired in a potential follow on mission that may launch the samples in an Orbiting Sample (OS) enclosure into orbit around Mars via a Mars Ascent Vehicle (MAV). The location for the execution of the BTC process would potentially be on-orbit about at an altitude of $500 \mathrm{~km}$. The OS would need to be contained and subjected to the BTC brazing S4 process and then inserted into an Earth Entry Vehicle (EEV) in an orbiting spacecraft.

The tests described above were performed using smaller scale containers. FEA analysis was done for both small scale containers to corroborate the analysis prediction with tests results and full scale containers. Towards the goal of testing full scale containers, a testbed has been developed with the intention to execute the BTC brazing automatically. For this purpose, a vacuum chamber with specially designed feedthroughs has been designed and procured. Mounting fixtures, control linear actuators, and force and temperature sensors were integrated inside the chamber. A design rendering view of the chamber and a photo of the vacuum chamber and the induction heating system are shown in Figure 4. Given that the chamber can reach pressure levels in the tens of micro-Torr vacuum, the use of argon for back-filling and avoiding oxidation has been eliminated from the test.

A custom machined coil with 2 turns was designed and procured and two options of cooling have been identified. These include a water cooled hollow tube coil and a high-precision machined solid Aluminum Nitride AlN ring for embedding the solid coil. The latter is currently in the process of being integrated into the chamber. This AlN heat sink (made by Sienna Technologies) has been specifically designed in segments that will encase the coil and keep the coil temperature below $150{ }^{\circ} \mathrm{C}[8]$. The two linear actuators control the preload of the lid and base double wall container halves and inner container separation step.

The process is monitored using two load cells, a pyrometer, and an optional infrared camera that provide force and temperature data to the controller of the S4 process. Photographs showing the pyrometer and a solid stainless steel ring to emulate the heating process are shown in Figure 5.

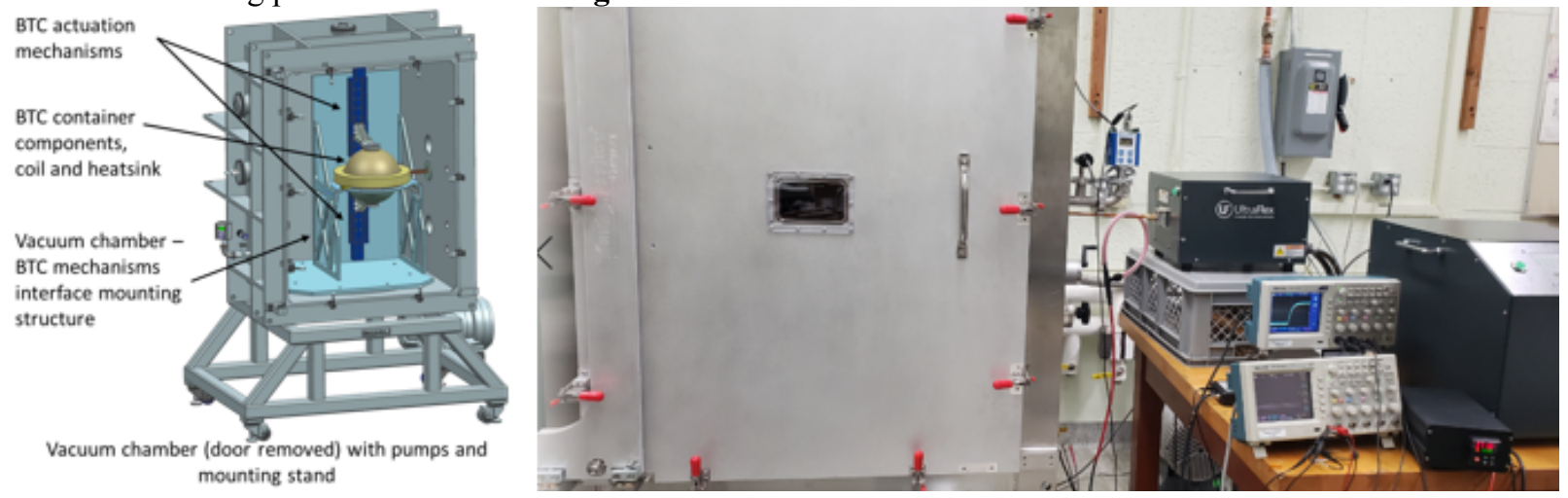

Figure 4: The brazing chamber prototype and its control hardware. Left: Design rendering, and Right: Photo of the hardware.

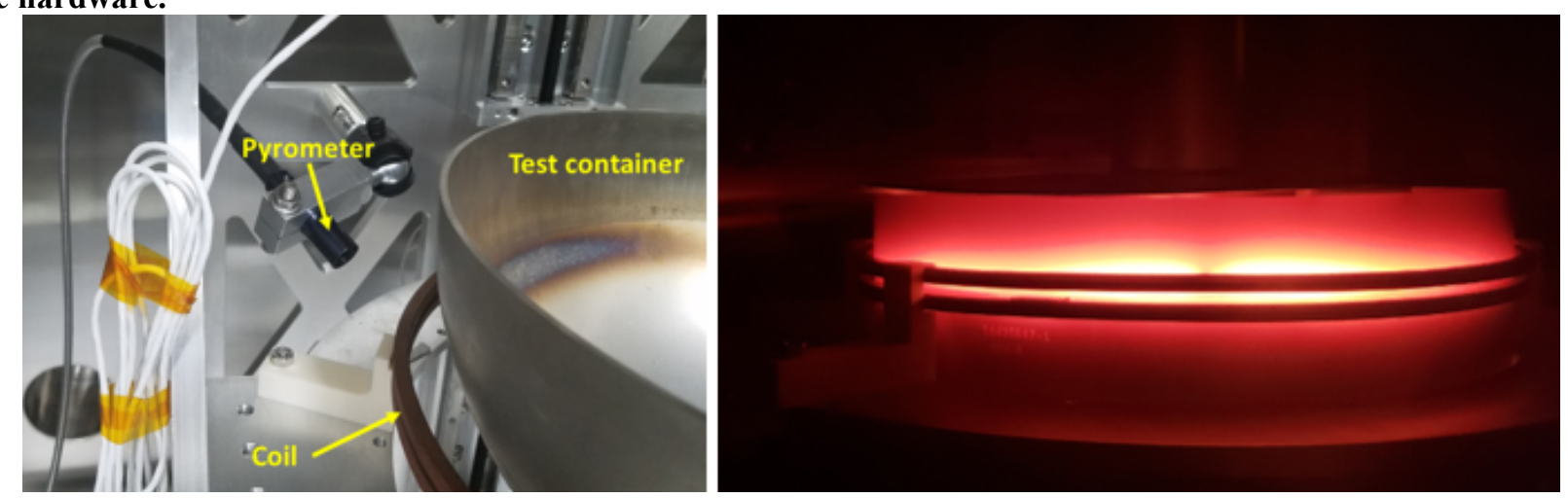

Figure 5: View of a test container inside the prototype chamber (left) and an induction heated test ring (right). 
Further development that is planned includes fabricating the full scale container and demonstrating the S4 process. In addition, various other container sizes and shapes are being analyzed to accommodate a smaller or larger number of samples. In addition, the OS shape is not fixed and may change from spherical to cylindrical with hemispherical end caps that would reduce the joint area diameter and hence the power consumption. A potential preliminary solid model rendering of the BTC configuration including the OS is shown in Figure 6.

\subsection{Summary and conclusions}

The potential return of samples from Mars and other planetary bodies would require protecting our planet from backward contamination. Such return missions would necessitate enclosing the samples inside a sealed container and the destruction or sterilization of any biological contaminants that may be present on the external surface before returning the container to Earth. To address the extremely strict planetary protection requirements, a brazing method has been developed and demonstrated that can meet the containerization at the current protection requirements [3 - 6]. The initial method of sterilization and sealing that was developed using a cylindrical configuration was found to have challenges related to potential jamming when doing the separation. A modification of the doublewall container design with a cone-in-a-cone braze surfaces has been demonstrated successful seaming, sealing and sterilization. The separation of the container from the launch vehicle either on the surface of the explored planet, on-orbit or even on Earth orbit can be accommodated by the technology. A double wall configuration is used with clean inner-spaces allowing brazing the inner container with the returned samples. For sealing the container, the use of brazing material that melts at temperatures higher than $500^{\circ} \mathrm{C}$ assures the destruction of biological materials via pyrolysis. The cone-withincone design has been conceived and demonstrated to allow a robotic mechanism to reliably handle and manipulate the components during the steps of the process.

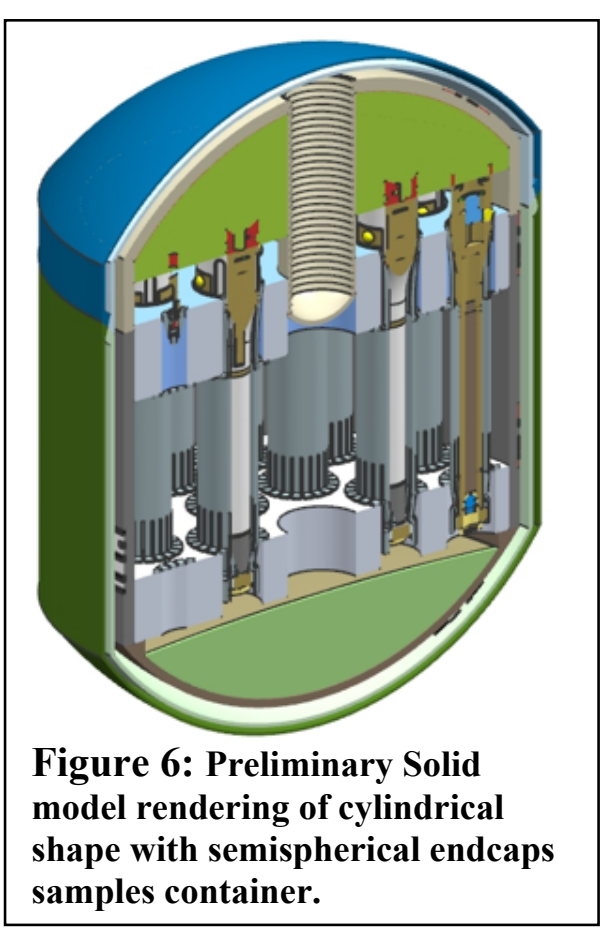

\subsection{Acknowledgment}

The decision to implement Mars Sample Return will not be finalized until NASA's completion of the National Environmental Policy Act (NEPA) process. This document is being made available for information purposes only.

Research reported in this manuscript was conducted at the Jet Propulsion Laboratory (JPL), California Institute of Technology, under a contract with National Aeronautics Space Administration (NASA). The authors would like to thank Robert Gershman and Morgan Henry, JPL, for their support of this reported study. Reference herein to any specific commercial product, process, or service by trade name, trademark, manufacturer, or otherwise, does not constitute or imply its endorsement by the United States Government or the Jet Propulsion Laboratory, California Institute of Technology.

\subsection{References}

[1] NRC Planetary Science Decadal Survey "Visions and Voyages" for 2013-2022 https://solarsystem.nasa.gov/2013decadal/

[2] Space Studies Board, (1998), Evaluating the Biological Potential in Samples Returned from Planetary Satellites and Small Solar System Bodies. National Research Council, National Academy Press, Washington, D.C., USA. 100 pages.

[3] Bar-Cohen Y., T. P. Rivellini, J. Wincentsen, and R. Gershman (April 6, 2004), "Simultaneous Separation, Seaming and Sealing using Brazing $\left(\mathrm{S}^{3} \mathrm{~B}\right)$ for Sample Containerization and Planetary Protection," NTR Docket No. 41024. 
[4] Bar-Cohen Y., A. K. Olorunsola, M. Badescu, X. Bao, and S. Sherrit, (September 25, 2007), "Simultaneous sealing and external surface sterilization of containers with samples," NTR Docket No. 45610.

[5] Bar-Cohen Y., X. Bao, H. J. Lee, M. Badescu, Z. Chang, S. Sherrit, and M. Burger, (July 21, 2014), "Mars Sample Return Sample Containment Using Brazing", Final Report, Mars Sample Return (MSR), Break the Chain of Contact (BTC) task.

[6] Badescu M., Y. Bar-Cohen, X. Bao, and B. Rhodes-Wickett, (April 6, 2016), "Using induction-heated brazing/soldering and double cone-within-cone for synchronous separation, sealing, seaming and external surface sterilization of containers with planetary samples", NTR Docket No. 50188.

[7] Schwartz M., (2003), Brazing $2^{\text {nd }}$ Edition ASM International, Materials Park, OH.

[8] Sherrit S., X. Bao, Y. Bar-Cohen, M. Badescu, and C. Lindsay, (submitted on September 8, 2017), "Solid State Cooling Ring (SSCR) (Induction Heating without Liquid Cooling the Coils)", NTR Docket No. 50623. Provisional Patent Application CIT 7883-P2 was filed by Caltech on Oct. 15, 2018. 